\title{
Rating the performance of a Brillouin distributed fiber sensor
}

\author{
Luc Thévenaz*, Marcelo A. Soto \\ EPFL Swiss Federal Institute of Technology, Institute of Electrical Engineering, \\ SCI STI LT, Station 11, CH-1015 Lausanne, Switzerland; \\ *E-mail: Luc.Thevenaz@EPFL.CH
}

\begin{abstract}
The effect of the characteristics of a generic distributed fiber sensor based on stimulated Brillouin scattering on the amplitude of the response is analyzed and a relation between the measurement noise on the time traces and the error on the determination of the Brillouin frequency shift is established. This makes possible to propose a simple figure-of-merit to fairly compare the performance of Brillouin distributed sensing systems.
\end{abstract}

Keywords: Stimulated Brillouin scattering, distributed optical fiber sensor, temperature sensing, strain sensing

\section{INTRODUCTION}

Tremendous progresses have been reported these past few years related to Brillouin distributed fiber sensing that turns into one of the most vivid field of research in optical fiber sensing. Thanks to advanced and ingenious techniques the spatial resolution has been improved by at least 2 orders of magnitude from meter to centimeter range ${ }^{1-7}$, while recent efforts have been carried out to extend the distance range from some $30 \mathrm{~km}$ to $120 \mathrm{~km}$ and more by using remote amplification $^{8-11}$ or smart pump pulse coding ${ }^{12-16}$. Among the numerous publications in the field it remains difficult to fairly evaluate the real progresses that are reported without an objective metric based on a rationale. For instance, how is it possible to compare between a sensor achieving a $2 \mathrm{~m}$ spatial resolution with a $2 \mathrm{MHz}$ Brillouin shift frequency accuracy over $100 \mathrm{~km}^{14}$, and a sensor showing a $3 \mathrm{~m}$ spatial resolution with $3 \mathrm{MHz}$ frequency accuracy over $120 \mathrm{~km}^{15}$ ? Is it a better performance than realizing a $5 \mathrm{~cm}$ spatial resolution over $5 \mathrm{~km}$ with $0.5 \mathrm{MHz}$ frequency accuracy ${ }^{6}$ ?

Currently there is no objective and simple way to compare these different results; for this reason, the purpose of this paper is to propose a figure-of-merit (FOM) calculated from a simple and universal modeling of a generic Brillouin distributed fiber sensor. Any progress brought by sophisticated and novel techniques should have the effect to make this FOM larger, so that the improvement can be really quantified.

The approach is based on one hand on the evaluation of the measurement contrast with distance and spatial resolution, which gives the usable signal amplitude, and on the other hand on the evaluation of the signal noise amplitude from the Brillouin frequency shift accuracy. Then the FOM is simply defined to be proportional to the signal-to-noise ratio, with some simple added considerations based on the limitations brought by the other nonlinear effects such as modulation instability and forward Raman scattering ${ }^{17}$ and by pump depletion ${ }^{18}$.

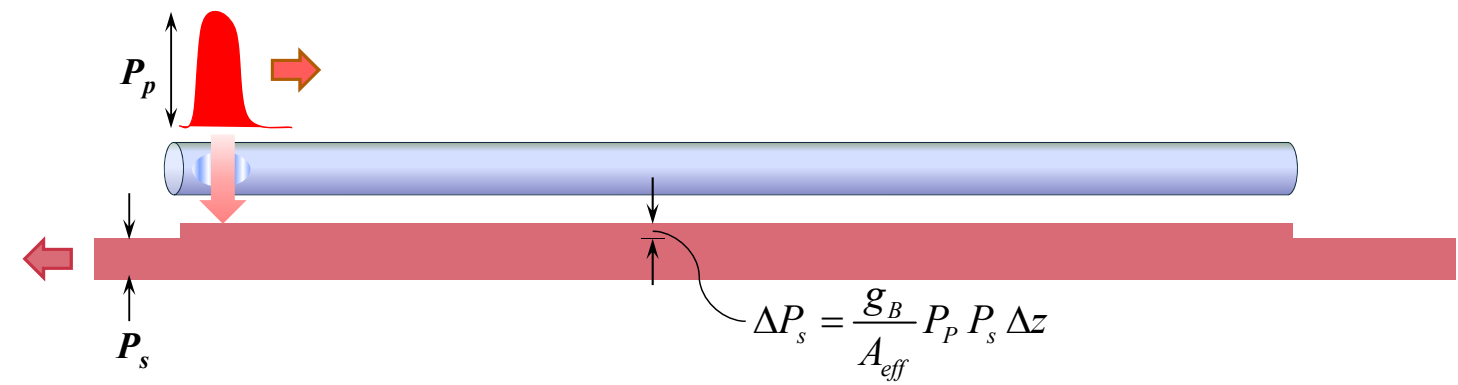

Figure 1. Interacting signals in a generic Brillouin time domain distributed fiber sensor. A light pulse of power $P_{P}-$ called pump - is interacting with a continuous wave of power $P_{s}$ - called probe or signal - through the intercession of an idler acoustic wave if a strict phase matching condition is satisfied. Phase matching depends on the frequency difference between pump and probe and gives rise to a local power transfer $\Delta P_{s}$ between the interacting optical waves.

OFS2012 22nd International Conference on Optical Fiber Sensors, edited by

Yanbiao Liao, Wei Jin, David D. Sampson, Ryozo Yamauchi, Youngjoo Chung, Kentaro Nakamura, Yunjiang Rao,

Proc. of SPIE Vol. 8421, 8421A7 · @ 2012 SPIE · CCC code: 0277-786/12/\$18 · doi: 10.1117/12.975290 


\section{SIGNAL GAIN AMPLITUDE}

In a generic Brillouin time domain distributed sensor the pump pulse interacts with the continuous wave (CW) probe through stimulated Brillouin scattering, as shown in Fig.1. Assuming for simplicity and without bringing restriction a gain configuration, i.e. the pump frequency is higher than the probe frequency, the amount of power transferred from the pump to the probe is given by:

$$
\Delta P_{s}(z)=P_{s}(z)\left[\mathrm{e}^{\frac{g_{B}}{A_{e f f}} P_{P}(z) \Delta z}-1\right]
$$

where $g_{B}$ represents the linear Brillouin gain coefficient, $A_{\text {eff }}$ the nonlinear effective area of the guided mode and $\Delta z$ the interaction length, equivalent to the spatial resolution and determined by half the pump pulse duration multiplied by the group velocity of the guided mode. This expression is valid for pulses longer than the acoustic amplitude lifetime $\tau=11 \mathrm{~ns}$, so that the stationary approximation for the acoustic wave can be used. It must be pointed out that advanced configurations based on the pre-excitation of the acoustic wave turn out to experience a power transfer that is also given by Equ.1, provided that the pre-excitation is long enough to let the acoustic wave reach its steady state value ${ }^{19}$, so that this expression can safely be used for all ranges of spatial resolution.

Assuming a small gain $\Delta P_{s}(z)<<P_{s}(z)$, observed in all practical and interesting situations, Equ.1 can be simplified as:

$$
\Delta P_{s}(z)=\frac{g_{B}}{A_{e f f}} P_{P}(z) P_{s}(z) \Delta z
$$

Then further assuming that the accumulated power transfer from the pump to the probe along the fiber is negligible necessary experimental condition to avoid systematic errors on the determination of the Brillouin frequency shift ${ }^{18}-$ this absence of depletion makes the pump simply experience the linear loss $\alpha$ and, keeping the small gain approximation, it can be straiforwardly demonstrated that the local gained power $\Delta P_{s}$ is independent on the position $z$ :

$$
\Delta P_{s}(z)=\frac{g_{B}}{A_{e f f}} P_{P i} \mathrm{e}^{-\alpha z} P_{s i} \mathrm{e}^{-\alpha(L-z)} \Delta z=\frac{g_{B}}{A_{e f f}} P_{P i} P_{s i} \mathrm{e}^{-\alpha L} \Delta z
$$

where $P_{P i}$ and $P_{s i}$ are the input pump and probe powers, respectively. This situation directly results from the counterpropagative situation of the interacting optical waves in stimulated Brillouin scattering, as shown in Fig.2.

Now, since the signal gain is measured at the fiber near end (position $z=0$ ), the transferred power described in Equ.3 (corresponding to the sensor response) must propagate from $z$ to 0 and therefore will experience linear loss as well, resulting in the following gain measured at the fiber near end:

$$
\Delta P_{s}^{0}=\Delta P_{s}(z) \mathrm{e}^{-\alpha z}=\frac{g_{B}}{A_{e f f}} P_{P i} P_{s i} \mathrm{e}^{-\alpha(L+z)} \Delta z
$$

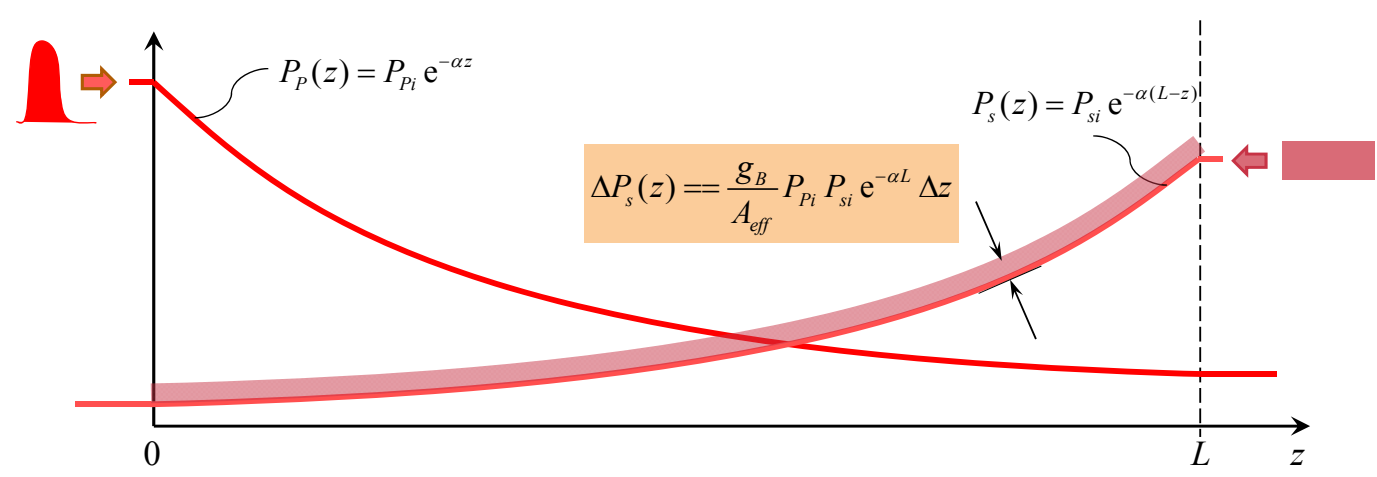

Figure 2. The counterpropagative interacting signals in a Brillouin time domain distributed fiber sensor experience linear loss and exponentially decay during their propagation. Since at each position $z$ the Brillouin response is proportional to the product the pump pulse of power $P_{P}(z)$ with the $\mathrm{CW}$ probe power $P_{s}(z)$ they show complementary amplitudes as a result of the counterpropagative situation and their product is invariant with position $z$. 
It is evident from this relationship that the observed sensor response will be smaller for large $z$ and thus the worst case corresponds to an interaction taking place close to the far fiber end at $z=L$. Thus performance must be proved with measurements performed at the far end where the sensor response is:

$$
\Delta P_{s}^{0}=\frac{g_{B}}{A_{e f f}} P_{P i} P_{s i} \mathrm{e}^{-2 \alpha L} \Delta z
$$

The effect of the attenuation turns out to be doubled when compared to a simple propagation; thus using standard values for the linear loss at $1550 \mathrm{~nm}$, increasing the sensing range by $7.5 \mathrm{~km}$ raises a difficulty similar to reducing the spatial resolution by a factor 2 . Note that the attenuation of the sensor response can be widely compensated by using amplification before detection, though with the penalty of the amplifier noise figure, but this amplification has to be eventually considered as a feature improving the figure-of-merit.

\section{IMPACT OF SIGNAL NOISE ON THE BRILLOUIN SHIFT ERROR}

In Brillouin distributed time-domain sensing the local Brillouin response is retrieved by extracting the value of the signal at a given position for each trace obtained sequentially by incrementing the frequency, so that a measurement of the local Brillouin gain spectrum is obtained. The peak gain frequency is then estimated by a fitting procedure and the accuracy will depend on the signal noise and on the number of points involved in the fitting procedure.

A standard approach consists in making a rough estimate of the peak frequency and of the peak gain value through a first pass, then keeping all experimental points above a given fraction of the peak value (e.g. 50\%) and performing a fit based on a limited number of degrees of freedom (e.g. parabolic) over the selected points.

Surprisingly, the estimation of the potential error on the Brillouin frequency shift for a given signal noise has been poorly addressed in the literature and only one relation has been given in a pioneering article on Brillouin fiber distributed sensing ${ }^{20}$, claiming an unusual fourth root dependence on the signal-to-noise ratio, without detailed justification and with no other dependence on other measurement conditions, such as the number of frequency points (or frequency sampling step).

In this work a new relation has been established directly following the procedure used for fitting, by calculating the propagation of errors on the parameters obtained from a least-square parabolic fit, subject to given and equal stochastic errors $\sigma$ on the measured gain spectral amplitudes (noise on the curve in Fig.3). For this calculation a normalized gain response has been assumed, i.e. the response is 0 in absence of gain (far from the resonance) and is 1 at the estimated peak response, and the noise amplitude $\sigma$ is also expressed as a fraction of this normalized response. If $\Delta v_{B}$ is the estimated FWHM width of the resonance and $\delta$ is the frequency sampling step (frequency increment between successive temporal traces), the variance on the estimation of the resonance central frequency becomes:

$$
\sigma_{v}^{2}=\frac{3}{2} \sigma^{2} \delta \Delta v_{B}
$$

This expression is valid if $\delta<<\Delta v_{B}$ (large number of sampling points) and if only data points with a normalized gain larger than 0.5 are considered. If this fraction is different, it just changes the multiplying constant on the right term. It must be pointed out that logically this expression gives a strict proportionality between signal noise and frequency error and the number of sampling points has a direct impact on the error of the estimation.

\section{FIGURE OF MERIT}

In Equ. 5 the pump and probe input powers can be considered as fixed for all types of sensors, since they are essentially given by the critical power for other nonlinear effects ${ }^{17}$ and by depletion ${ }^{18}$, respectively. These two effects are actually scaled by the effective nonlinear length of the fiber $L_{e f f}=\left(1-\mathrm{e}^{-\alpha L}\right) / \alpha$ that tends towards $\alpha^{-1}$ for very long fibers. It means that for short fibers $\left(\mathrm{L}<\alpha^{-1}\right)$ pump and probe powers can be raised in the same proportion to eventually enhance 
the sensor response. This feature has to be considered for a fair comparison between short high spatial resolution sensors and long distance systems. The number $N$ of averaging performed on the time traces has also to be considered, since the noise can be arbitrarily decreased proportionally to $\sqrt{N}$ at the expense of a longer acquisition time.

Using these considerations and Equ.5-6, the following figure of merit is defined for distributed Brillouin fiber sensors:

$$
F O M=\frac{\left(\alpha L_{e f f} \mathrm{e}^{\alpha L}\right)^{2}}{\Delta z \sqrt{N}} \frac{\sqrt{\delta \Delta v_{B}}}{\sigma_{v}}
$$

With consistent units and $\Delta z$ normalized to $1 \mathrm{~m}$, this FOM turns out to be 1 for a typical commercial sensor of length $L=21 \mathrm{~km}$, of spatial resolution $\Delta z=1 \mathrm{~m}$ and of error on the Brillouin frequency $\sigma_{v}=1 \mathrm{MHz}$, obtained with $N=1024$ temporal trace averaging and with frequency sampling step $\delta=1 \mathrm{MHz}$, in a standard fiber with $\Delta v_{B}=27 \mathrm{MHz}$ and a $0.2 \mathrm{~dB} / \mathrm{km}$ attenuation $\left(\alpha^{-1}=22 \mathrm{~km}\right)$.A comparison with past published results in the literature remains yet speculative, since actually no publication fully informs about all experimental parameters necessary to calculate this figure of merit. The authors wish that this expression will stimulate the community to inform enough about their experimental conditions to calculate the FOM and generate emulation for achieving real progress and milestones in this promising field.

\section{REFERENCES}

[1] Hotate, K., "Measurement of Brillouin gain spectrum distribution along an optical fiber using a correlation-based techniqueproposal, experiment and simulation", IEICE Transactions on Electronics E83-C(3), 405-411 (2000)

[2] Bao, X., Brown, A., DeMerchant, M. and Smith, J., "Characterization of the Brillouin-loss spectrum of single-mode fibers by use of very short (<10-ns) pulses", Opt. Lett. 24(8), 510-512 (1999)

[3] Kishida, K. and Li, C.-H., "Pulse pre-pump-BOTDA technology for new generation of distributed strain measuring system", in Structural Health Monitoring and Intelligent Infrastructure, pp. 471-477, Taylor \& Francis Group, London (2006).

[4] Brown, A. W., Colpitts, B. G. and Brown, K., "Dark-Pulse Brillouin Optical Time-Domain Sensor With 20-mm Spatial Resolution", J. Lightwave Technol. 25(1), 381-386 (2007)

[5] Li, W., Bao, X., Li, Y. and Chen, L., "Differential pulse-width pair BOTDA for high spatial resolution sensing", Opt. Express 16(26), 21616-21625 (2008)

[6] Foaleng, S. M., Tur, M., Beugnot, J. C. and Thevenaz, L., "High Spatial and Spectral Resolution Long-Range Sensing Using Brillouin Echoes", Journal of Lightwave Technology 28(20), 2993-3003 (2010)

[7] Song, K. Y., Chin, S., Primerov, N. and Thevenaz, L., "Time-Domain Distributed Fiber Sensor With $1 \mathrm{~cm}$ Spatial Resolution Based on Brillouin Dynamic Grating", Journal of Lightwave Technology 28(14), 2062-2067 (2010)

[8] Alahbabi, M. N., Cho, Y. T. and Newson, T. P., "150-km-range distributed temperature sensor based on coherent detection of spontaneous Brillouin backscatter and in-line Raman amplification", J. Opt. Soc. Am. B 22(6), 1321-1324 (2005)

[9] Rodriguez-Barrios, F., Martin-Lopez, S., Carrasco-Sanz, A., Corredera, P., Ania-Castanon, J. D., Thevenaz, L. and GonzalezHerraez, M., "Distributed Brillouin Fiber Sensor Assisted by First-Order Raman Amplification", Lightwave Technology, Journal of 28(15), 2162-2172 (2010)

[10] Martin-Lopez, S., Alcon-Camas, M., Rodriguez, F., Corredera, P., Ania-Castañon, J. D., Thévenaz, L. and Gonzalez, M., "Brillouin optical time-domain analysis assisted by second-order Raman amplification", Opt. Express 18, 18769-18778 (2010)

[11] Angulo-Vinuesa, X., et al, "Raman-Assisted Brillouin Distributed Temperature Sensor over $100 \mathrm{~km}$ featuring $2 \mathrm{~meter}$ Resolution and $1.2^{\circ} \mathrm{C}$ Uncertainty", Journal of Lightwave Technology 30(8), 1060-1065 (2012)

[12] Soto, M. A., Bolognini, G., Di Pasquale, F. and Thévenaz, L., "Simplex-coded BOTDA fiber sensor with $1 \mathrm{~m}$ spatial resolution over a $50 \mathrm{~km}$ range", Opt. Lett. 35(2), 259-261 (2010)

[13] Soto, M. A., Bolognini, G. and Di Pasquale, F., "Analysis of pulse modulation format in coded BOTDA sensors", Opt. Express 18(14), 14878-14892 (2010)

[14] Dong, Y., Chen, L. and Bao, X., "Time-division multiplexing-based BOTDA over 100km sensing length", Opt. Lett. 36(2), 277$279(2011)$

[15] Soto, M. A., Bolognini, G. and Pasquale, F. D., "Long-range simplex-coded BOTDA sensor over 120km distance employing optical preamplification", Opt. Lett. 36(2), 232-234 (2011)

[16] Soto, M. A., Taki, M., Bolognini, G. and Di Pasquale, F., "Optimization of a DPP-BOTDA sensor with $25 \mathrm{~cm}$ spatial resolution over $60 \mathrm{~km}$ standard single-mode fiber using Simplex codes and optical pre-amplification", Opt. Express 20, 6860-6869 (2012)

[17] Foaleng, S. M. and Thevenaz, L., "Impact of Raman scattering and modulation instability on the performances of Brillouin sensors", 21st International Conference on Optical Fiber Sensors, 7753, 77539V (2011)

[18] Thevenaz, L., Foaleng Mafang, S. and Lin, J., "Impact of pump depletion on the determination of the Brillouin gain frequency in distributed fiber sensors", 21st International Conference on Optical Fiber Sensors, 7753, 775322 (2011)

[19] Beugnot, J.-C., Tur, M., Foaleng Mafang, S. and Thévenaz, L., "Distributed Brillouin sensing with sub-meter spatial resolution: modeling and processing", Opt. Express 19(8), $7381-7397$ (2011)

[20] Horiguchi, T., Shimizu, K., Kurashima, T., Tateda, M. and Koyamada, Y., "Development of a distributed sensing technique using Brillouin scattering", Journal of Lightwave Technology 13(7), 1296-1302 (1995) 